\title{
Exploring underwater target detection by imaging polarimetry and correlation techniques
}

\author{
M. Dubreuil, ${ }^{1}$ P. Delrot, ${ }^{1}$ I. Leonard, ${ }^{1}$ A. Alfalou, ${ }^{1, *}$ C. Brosseau ${ }^{2}$ and A. Dogariu ${ }^{3}$ \\ ${ }^{1}$ Vision-L@BISEN, ISEN Brest, 20 rue Cuirassé Bretagne, CS 42807, \\ 29228 Brest Cedex 2, France \\ ${ }^{2}$ Université Européenne de Bretagne, Université de Brest, Lab-STICC,6 avenue Le Gorgeu, \\ CS 93837, 29238 Brest Cedex 3, France. \\ ${ }^{3}$ CREOL, The College of Optics and Photonics, University of Central Florida, \\ P.O. Box 162700, Orlando, FL, USA
}

*Corresponding author: ayman.al-falou@isen.fr

Underwater target detection is investigated by combining active polarization imaging and optical correlation-based approaches. Experiments were conducted in a glass tank filled with tap water with diluted milk or seawater and containing targets of arbitrary polarimetric responses. We found that target estimation obtained by imaging with two orthogonal polarization states always improves detection performances when correlation is used as detection criterion. This experimentally study illustrates the potential of polarization imaging for underwater target detection and opens interesting perspectives for the development of underwater imaging systems.

(C) 2012 Optical Society of America

OCIS codes: 110.0113,110.5405, 100.5010. 


\section{Introduction and background}

Underwater imaging is a challenging problem because of the intrinsic optical properties of water, dissolved organic and inorganic matter, and suspended particles. Light attenuates exponentially as it travels through water due to absorption and scattering (both forward and backward). Forward scattered light is reflected by an object and then is diffused back to the detector. It generally produces blurred images. Backscattered light is reflected by particles back to the detector before reaching the object. It has for main effect to degrade the contrast of immerged objects because of the veil that superimposes on the image. In addition, light absorption is wavelength-dependent (shorter wavelengths travel longer), which makes the images be dominated essentially by blue color. As a result questions remain whether one can overcome these issues. For example, image processing methods such as image restoration or image enhancement have been developed [1,2]. However, image restoration requires many model parameters that can be extremely variable depending on the target's spatial position and water constitution. Consequently, it can be difficult to use in real situations. Image enhancement techniques do not rely on any physical model but aim at producing visually pleasing images. One drawback of these methods is that they are spatially invariant. They have the advantage that they are based on commonly used computer vision techniques. Hence, image enhancement methods are simpler and faster of use than restoration methods.

Previously, it has been shown that it is possible to use color information for underwater detection of objects [2]. Specifically, Beer-Lambert law permits to define underwater colors and attenuation coefficients depending on observation distance and illumination. It is noteworthy that this method permits to identify a specific object in underwater conditions [2]. However, it requires much larger computation time and resources compared to the method which is 
developed in this work. Additionally, the method developed in Ref. [2] requires an a priori knowledge of the color of the underwater object and does not permit discrimination of two objects having similar colors.

Many underwater imaging systems based on range-gating (time discrimination), laser line scanning (spatial discrimination), structured lightning, modulation/demodulation techniques, multiple perspective image construction, and polarization discrimination have also attracted significant interest $[3,4]$. Polarization filtering is adapted to underwater target detection and identification [6-20]. In this context, combining polarization filtering and a correlation technique is justified because: (i) it is simple to implement, (ii) it is compatible with polarization filtering, and (iii) it allows us for simultaneous detect and identify target objects.

A basic issue in the field of underwater mine warfare concerns the development of compact, low-power-consumption, and high-speed underwater vehicles. A recent study [5] reported that optical correlation-based methods can be used for automatic underwater target detection. This study is based on visibility enhancement by image pre-processing and generation of a large reference database of target. Pushing back the limits of target recognition remains a clear challenge. Thus, developing image acquisition is a prerequisite for improved underwater target detection. For that purpose, polarimetric imaging is the ideal technique since it is simple to implement, needs small power, and can be used for real-time applications. In addition, it allows increasing the contrast of the target images and decreasing both illumination effects and backscattering phenomena.

Here, the focus is on active polarization imaging and optical correlation-based methods to experimentally characterize underwater target detection. Experiments are conducted in a glass tank filled either with a scattering medium, or seawater. We perform active polarization imaging 
to suppress the veil in the scene whereas correlation is used for detection criterion and target localization.

The paper is organized as follows. Section 2 describes the use of polarization in underwater experiments. Section 3 describes the optical correlation-based method used for target detection. Section 4 presents the setup for experiment. The results of this analysis are described and discussed in Sec. 5. Finally conclusions are drawn in Sec. 6.

\section{Underwater polarization optics}

The behavior of polarized light in seawater is a topic attracting widespread attention [6-9]. Kouzoubov [6] reviewed experimental data and methods for calculating the Mueller matrix of sea water. Voss and Fry [7] were among the first to report angle-resolved Mueller matrix measurements of seawater. This study demonstrated that these matrices are generally consistent with Rayleigh-scattering regime by small particles. But only weak differences due to asymmetric particle shapes can be found using numerical simulations. The study of underwater polarized light led to the development of passive and active polarimetric imaging systems for improved target detection. Passive imaging uses ambient illumination from sunlight (or a cloudy sky) which is generally unpolarized. Light which has not reached the object becomes partially polarized by scattering and can be filtered by an analyzer placed in front of the detector $[9,10]$. However, natural illumination is inapplicable to dark environments where active imaging is preferred. It requires artificial illuminating systems and adapted detection schemes.

For active polarimetric imaging systems, several studies have proposed to exploit the

difference in polarimetric responses between backscatter and target. A full Mueller matrix imaging system in scattering medium was considered in Refs. [11-13]. By numerical 
simulations, it was found that specific combinations of Mueller matrix elements lead to better contrast between a target and its background than ordinary radiance [11]. This was experimentally verified by Lewis [13]. In [12], the authors measured Mueller matrices of several underwater targets. However, full Mueller matrix measurements require considerable data acquisitions and are therefore time consuming. Recent studies [14-16] have pointed out that the strong backscatter created by artificial illumination can be reduced by performing a single acquisition with adapted input and output polarization states. For example, Gibert and Pernicka [14] showed that using circularly polarized light permits reduction of the backscatter and increase underwater visibility. Based on the assumptions of totally depolarizing target and change of handedness of the circularly polarized state after reflection by scattering particles, the contrast of the target should be improved. Their experimental results led to an increase by a factor of two of the visibility range with circular polarization measurements compared to intensity measurements. In a recent study, Bartolini and co-workers [15], showed that using a cross-linear polarization detection scheme enables an increase of about two attenuation lengths in the transmission capacities of a modulating retroreflector. They take advantage of the fact that linear polarization is mainly preserved in the backscatter signal. Mullen and co-workers [16] confirmed that imaging in the cross polarized state using linearly polarized light and in the copolarized state using circularly polarized light increases the precision of phase measurements in amplitude modulated laser experiments. Other studies [17-20] have demonstrated that performing measurements with two orthogonal states permits decrease of the backscattering effect. This arises because a substantial part of the backscatter is still present in the orthogonal state image. As the co-polarized image gives insight into the shape of the backscatter, the suggested idea is to subtract a fraction of the co-polarized image from the opposite-polarized image in order to completely remove it. 
Since we adopt Schechner's approach [20] that deals with arbitrary polarimetric response a brief discussion of this algorithm is in order. Underwater images are composed of the object signal, the forward scattered signal, and the backscattered signal [21]. We ignore the forward scatter part of the total signal which is responsible for image blurring. Thus the measured image $I(x, y)$ is

$$
I(x, y)=S(x, y)+B(x, y),
$$

where $S(x, y)$ is the target signal, and $B(x, y)$ corresponds to the backscatter. A cross-linear (circular) image is obtained with a given input polarization illumination and a detection in the orthogonal state. A co-linear (circular) image is also obtained with detection in the same state as the illumination. These two images, namely $I_{\min }$ and $I_{\max }$, are acquired, corresponding respectively to the cross-linear (co-circular) image and the co-linear (cross-circular) image. Since an important fraction of linear (circular) polarization is preserved (reversed) upon backscattering at large angles, imaging in the cross linear (co-circular) state gives an image with less backscatter and target signal. The images are expressed as

$$
\begin{aligned}
& I_{\text {max }}(x, y)=S_{\text {max }}(x, y)+B_{\text {max }}(x, y) \\
& I_{\text {min }}(x, y)=S_{\text {min }}(x, y)+B_{\text {min }}(x, y) .
\end{aligned}
$$

The degree of polarization (DOP) of both target and backscatter can be described as

$$
p_{\text {targ }}(x, y)=\frac{S_{\text {max }}(x, y)-S_{\text {min }}(x, y)}{S_{\text {max }}(x, y)+S_{\text {min }}(x, y)},
$$

and 


$$
p_{\text {scat }}(x, y)=\frac{B_{\max }(x, y)-B_{\min }(x, y)}{B_{\max }(x, y)+B_{\min }(x, y)} .
$$

The target signal and the backscatter can be expressed as

$$
S(x, y)=S_{\max }(x, y)+S_{\min }(x, y),
$$

and

$$
B(x, y)=B_{\max }(x, y)+B_{\text {min }}(x, y),
$$

which lead to the expression for the target estimation $\hat{S}$ and the backscatter estimation $\hat{B}$

$$
\hat{S}=\frac{1}{p_{\text {scat }}-p_{\text {targ }}}\left[I_{\text {min }}\left(1+p_{\text {scat }}\right)-I_{\text {max }}\left(1-p_{\text {scat }}\right)\right],
$$

and

$$
\hat{B}=\frac{1}{p_{\text {scat }}-p_{\text {targ }}}\left[I_{\text {max }}\left(1-p_{\text {targ }}\right)-I_{\text {min }}\left(1+p_{\text {targ }}\right)\right] \quad .
$$

The target estimation can thus be deduced from two measurements, i.e. $I_{\min }$ and $I_{m a x}$, given the value of $p_{\text {scat }}$, because $p_{\text {targ }}$ only contributes to a scale factor to the signal reconstruction. In the following, the target estimation will always be calculated from Eq.(8) assuming $p_{\text {targ }}=0$. It is worth noting that the value of $p_{\text {scat }}$ is critical to our analysis and needs to be evaluated precisely. For that purpose, it is necessary to find in a region in the image with no target to estimate $p_{\text {scat }}$. In our experiments, it is calculated by averaging the DOP values in the designated region displayed as the red square in Fig. 3(a),. 
Schechner's analysis is general and can be applied with linearly as well as circularly polarized light. In these earlier studies optical thickness was assumed to be less than 10 . Consequently, the polarization memory effect [22] is irrelevant for seawater.

Based on the above survey, we conclude that studies on underwater target detection often quantify the improvement supplied by polarization by comparing the contrast between the target and background in intensity imaging mode and that obtained with polarized light. Other parameters are defined to assess image quality, include sometimes subjective criteria [23]. But, these parameters are not well suited to automatic target detection. On the contrary, optical correlation-based approaches can perform a comparison between a target image and a reference image, and are fully consistent with automatic target recognition and localization. The primary aim of this work is to quantify the improvement supplied by polarization imaging by using correlation as the detection criterion.

\section{Optical correlation}

In this work we propose a procedure which combines polarization imaging and an optical correlation-based method to remove backscatter signal and enhance the target detection in the scene. On the one hand, this procedure crucially relies on the compatibility of optical correlation methods with this experimental approach. On the other hand, it has been reported in several studies [26-33] that the correlation method employed has good detection and identification performances. However, due to practical reasons, only a numerical implementation of the correlator was considered. Optical correlation techniques have generated considerable interest in recent years to develop automatic target recognition systems [24-25], and face recognition algorithms [26]. New correlation filters [26-27] and metrics for decision criteria [28-29] were 
proposed to increase robustness and/or discriminating capacities. Moreover, numerical correlation techniques were compared to independent component analysis based approaches for biometric recognition applications [30]. The advantage of using correlation relies on the possibility to create multiple-reference composed filters allowing us to increase robustness and speed [31]. Recently, electronic implementation of correlation filters on field programmable gate array [32] and graphics processing unit [33] give the possibility to perform numerically correlation in real time with good performance and less difficulties than optically.

The principle of image correlation is to compare a target image and a reference one. This results in a more or less intense peak in the correlation plane, depending on the degree of resemblance between the target and the reference images. The location of the correlation peak also gives the location of the target in the image. Two architectures are of particular interest: the Vanderlugt correlator (VLC) [34] and the joint transform correlator (JTC) [35]. The former is preferred for object identification whereas the latter is more suited for object tracking. Figure 1 shows the synoptic diagram of VLC architecture used in this study. It is based on the multiplication of the spectrum of the target image by a correlation filter $H$ fabricated from a reference image. An inverse Fourier transform is then performed which give the correlation plane.

Much research has been done to create robust and/or discriminating filters, see, e.g. [26] and references therein. In this study, we use the phase-only filter (POF), which is defined as:

$$
H(\mu, v)=\frac{R^{*}(\mu, v)}{|R(\mu, v)|},
$$

where $\mu$ and $v$ are the coordinates in the frequency plane, $R(\mu, v)$ is the spectrum of the reference image and $*$ denotes the complex conjugate. POF is used instead of a matched filter because it is 
highly discriminating, i.e. the false alarm rate is strongly decreased. In this work, a segmented composite filter developed in Refs. [26-27] is used to increase the robustness of POF. This filter is built by including several reference images corresponding, e.g. to different orientations and/or scales of the target [26]. Thus, our filter represents a good compromise between the properties of discrimination and robustness.

The criterion used for target recognition is the peak-to-correlation energy, which is defined as follows:

$$
P C E=\frac{\sum_{i, j}^{M} E_{\text {peak }}(i, j)}{\sum_{i, j}^{N} E_{\text {correlation plane }}(i, j)},
$$

where $E$ is the intensity of the correlation plane, $N$ is the size of the correlation plane and $M$ is the size of the correlation peak ( 9 pixels around the maximum were considered here). This criterion evaluates energy contained in the correlation peak compared to energy contained in the correlation plane. A high value of the PCE means a strong resemblance between the target and the reference image. A threshold value for the PCE can thus be chosen which is well suited for automatic target detection. The degree of similarity between the target object and the reference image is related to the height and width of the correlation peak. Good similarity is associated with a narrow and high correlation peak. However, the latter is not localized on a single pixel. In the current study dealing with noisy data we define the energy within the correlation peak as the energy around the correlation peak up to a $3 \mathrm{~dB}$ height. This corresponds to a 9 pixel 
neighborhood around the correlation peak. Other criteria could have been chosen to determine the degree of similarity such as in Ref.[29].

\section{Experimental setup}

The experimental setup used for underwater target detection is depicted in Fig. 2. The source is a He-Ne Laser emitting at $632.8 \mathrm{~nm}$. The spatial coherence of the laser beam is scrambled by a rotating diffuser (BFI Optilas) in order to avoid speckle patterns. The beam is then expanded to illuminate the scene composed of a suspended immersed target. We used linear polarizers purchased from Newport and precision quarter wave plates (QWPs) obtained from Meadowlark Optics. All optical elements were antireflection-coated at $633 \mathrm{~nm}$. The camera is a 14 bits digital CCD camera (Stingray F-033, AVT) with a $656 \times 492$ resolution. The field of view for the experiments was $5 \mathrm{~cm} \times 5 \mathrm{~cm}$. Input and output polarization states were selected with proper orientation of the polarizers and the QWPs. Linear polarization states (horizontal and vertical) are obtained by removing the QWPs and circular polarization states (right and left) are obtained by placing the QWPs at $\pm 45^{\circ}$ to the polarizers. The detection angle was set to $\theta=10^{\circ}$ for the experiments.

We used a glass tank filled with a scattering medium composed of $10 \mathrm{~L}$ of tap water and semi-skimmed or skimmed milk. Milk is composed of spherical particles of different size (casein molecules $\sim 0.04-0.3 \mu \mathrm{m}$ and fat globules $\sim 1-20 \mu \mathrm{m}$ ). It has been a long-time that scattering experiments have suggested that milk can mimic the scattering properties of sea water. While semi-skimmed milk contains more fat globules resulting in a Mie scattering regime skimmed milk is mostly composed of small particles resulting in a dominant Rayleigh scattering regime. 
The scattering coefficient $\mu_{s}$ for skimmed milk and semi-skimmed milk are $0.42 c \mathrm{~cm}^{-1}$ and $1.40 \mathrm{~cm}^{-1}$, respectively, where $c$ is the concentration of milk in water [36]. The attenuation length (or optical thickness) $\tau_{0}$ is defined as the product of the scattering coefficient $\mu_{s}$ by the physical depth $d$, so that $\tau_{0}=\mu_{s} d$. For the wavelength considered here, the absorption coefficient of milk can be safely ignored with respect to the scattering coefficient. Extinction coefficient data ranging between 0.1 and $4 \mathrm{~m}^{-1}$ were reported earlier for seawater. Milk concentration was adjusted to obtain extinction coefficient in this range. In realistic environment, targets have different reflectance and polarimetric responses [37]. Three targets with different reflectance and polarimetric responses were used, i.e. a plastic plug ( $\mathrm{DOP}=0.1)$, a painted metal $(\mathrm{DOP}=0.3)$, and

a rusted metal $(\mathrm{DOP}=0.9)$. Calculations were made with $p_{\text {targ }}=0$, because $p_{\text {targ }}$ only contributes to a scale factor to the reconstructed signal image, i.e. Eq.(8). Several acquisitions were sequentially taken: intensity image (with no polarizers) denoted hereafter I, co- and crosslinearly polarized light respectively abbreviated COL and CRL, and co- and cross-circularly polarized light respectively abbreviated COC and CRC. The signal estimations were then calculated from Eq.(8) for linearly and circularly polarized lights.

\section{Results and discussion}

The panels of Fig. 3 show images of the plastic plug for $20 \mathrm{ml}$ of skimmed milk $\left(\mu_{\mathrm{s}}=0.08 \mathrm{~cm}^{-1}\right.$, scattering mean free path $\left.1_{\mathrm{s}}=12.5 \mathrm{~cm}\right)$. Typically, the distance that light travels before reaching the target is $15 \mathrm{~cm}$, corresponding to an optical thickness of 1.2. Images taken with I and CRL modes, linear estimation, and in pure water are presented. Differences between these images merit discussion. 
It is interesting to observe that the backscatter clearly appears in the intensity image (Fig. 3(a)) in its left part. Another interesting feature is that the contours of the plastic plug are not well defined in the intensity image. In the CRL image shown in Fig. 3(b), most of the backscatter is suppressed. We observe that the backscatter has the same polarization as the incident polarized light so imaging in the opposite polarization mode is a way to limit the backscatter. However, the backscatter is not fully polarized and a substantial part of it is still present in the CRL image. In the estimation signal, i.e. Eq.(8), the backscatter is totally removed and the image is very close to the image obtained in pure water. Based on these observations, it appears that performing two measurements with two orthogonally polarized states leads to better performance than a single measurement. A photograph of the plastic plug is shown in Fig. 3(e).

Fig. 4 shows a comparison between correlation planes and PCE for each image shown in Fig. 3. The reference image is taken as the image in clear water, i.e. Fig. 4(d), leading to the autocorrelation plane. Note that we consider normalized correlation planes with respect to the peak height in Fig. 4 and PCE values are multiplied by a factor of $10^{4}$

We point out that Fig. 4(a) shows a very noisy correlation plane because the backscatter creates additional contours that correlate with the reference image ones. Consequently, PCE is small. The correlation plane looks better in the CRL image (Fig. 4(b)), and even better for the target estimation (Fig. 4(c)). The PCE parameter provides valuable information about images and can be useful for automatic target recognition.

In order to validate the advantage supplied by polarization imaging, several experiments were conducted with varying milk concentration and for three different targets (plastic plug, painted and rusted metal). Figure 5 illustrates that as the optical thickness increases in skimmed and semi-skimmed milk, the PCE tends to decrease for I, COL and CRL, COC and CRC imaging modes, and target estimations for linear (ESTL) and circular (ESTC) polarizations. It should be 
noted that the PCE values are normalized by the highest value for one set of experiment (corresponding to a specific target). Hence, these values can safely be compared for different polarizations states.

We find that all curves have the same behavior. For depolarizing media (plastic plug and painted metal) the PCE values (and thus the detection efficiency) are lower for COL and CRC than for I. Imaging with such polarization modes does not allow for backscatter suppression. Moreover, a part of the target signal is filtered with these modes. The PCE is higher for COC and CRL because of backscatter suppression. Larger PCEs are obtained for ESTL and ESTC for which full backscatter is suppressed. For polarization-maintaining medium (rusted metal) one should note that imaging with COC and CRL is not beneficial because a large part of the target signal is filtered as well as the backscatter. However, target estimation leads to better detection efficiency than for I, CRC, and COL. We note also that no significant differences can be seen between imaging with circularly and linearly polarized lights. We further find that higher optical thickness can be achieved in the case of semi-skimmed milk. This agrees with the fact, in Mie scattering regime, the backscatter is smaller than in Rayleigh scattering regime, and thus it requires more milk concentration to obtain significant veil.

An important issue when dealing with target estimation is the value of $p_{\text {scat }}$. Figure 6 shows $p_{\text {scat }}$ as a function of optical thickness for experiments using skimmed milk.

As one can see, $p_{\text {scat }}$ increases slightly at small optical thickness. This behavior is due to the small amount of backscatter which is almost non uniform over the averaging area. For optical thickness ranging between 0.2 and $0.4, p_{\text {scat }}$ reaches its highest value $(\cong 0.8)$. If optical thickness is further increased, $p_{\text {scat }}$ progressively decreases because of the increasing number of multiply scattered photons collected by the detector. One should keep in mind that these values strongly 
depend on the detector field of view [16]. As the signal estimation is very sensitive to $p_{\text {scat }}$ and because it is difficult to predict its evolution, the detection scheme must include a specific measure of $p_{\text {scat }}$ for each image. It requires selecting an area with no target but only the backscatter.

To gain more insight with realistic environment of target detection, e.g. underwater mine, experiments using turbid seawater (from Brest Bay area) and river water (Penfeld, Brest, France) were conducted. Painted metal like that used in experiments with milk is considered as the target. As the turbidity of the water is fixed target was progressively moved to vary optical thickness. Figure 7 (a) and (b) present PCE as a function of target depth for respectively seawater and lake water. The reference images are the painted metal in pure water taken at different depths.

Likewise, our analysis suggests that adapted polarization imaging (CRL and COC) improves target detection compared to intensity imaging. Target estimation is the best way to achieve good detection when turbidity of the embedding medium is large. As turbidity ofwater is fixed and given the dimensions of our glass tank smaller optical thicknesses can be achieved in these experiments compared to our previous experiments with diluted milk. The turbidity of the lake water was found to be much smaller than that of seawater.

\section{Summary}

In summary, we find that polarization imaging associated to an optical correlation-based method as a decision criterion can improve underwater target detection. In case of a depolarizing object, performing one measurement using adapted polarization states (cross-linear or co-circular) leads to better performance than intensity imaging because it filters the major part of the backscatter and only a small part of the target signal. For polarization-maintaining objects, performing only 
one measurement is insufficient to improve target detection because an important part of the signal is lost. However, whatever the target is, performing two measurements with two orthogonal polarization states improves recovery of the target signal by suppressing the backscatter that veils the object. The contours of the target are thus well defined which enhances correlation performance. Experiments performed in different scattering media and different targets showed that there is always an improvement in target detection when target estimation is considered. No difference in decision performance was found between linear and circular polarized lights. Although the two measurements required for signal estimation were obtain sequentially, it would be interesting to use recent progress made in snapshot polarization imaging [38,39] to obtain these measurements instantaneously.

\section{Acknowledgments}

The authors thank J. Corbel for technical assistance and A. Arnold Bos for fruitful discussion. 


\section{References}

1. R. Schettini and S. Corchs, "Underwater image processing: state of the art of restoration and image enhancement methods", EURASIP J. Advances in Signal Processing, 746052 (2010), doi:10.1155/2010/746052.

2. S. Bazeille, I. Quidu, and L. Jaulin, "Color-based underwater object recognition using water light attenuation”, Intelligence Service Robotics 5, 109-118 (2012)

3. D. M. Kocak, F. R. Dalgleish, F. M. Caimi, and Y. Y. Schechner, "A focus on recent developments and trends in underwater imaging”, Mar. Technol Soc. J. 42, 52-67 (2008).

4. F. Bonin, A. Burguera, and G. Olivier, "Imaging systems for advanced underwater vehicles", J. Mar. Res. 13, 65-86 (2011).

5. I. Leonard, A. Arnold-Bos, and A. Alfalou, "Interest of correlation-based automatic target recognition in underwater optical images: theoretical justification and first results", Proc. of SPIE 7678, 767800 (2010).

6. A. Kouzoubov, M. J. Brennan, and J. C. Thomas, "Treatment of polarization in laser remote sensing of ocean water”, Appl. Opt. 37, 3873-3885 (1998).

7. K. J. Voss and E. S. Fry, "Measurement of the Mueller matrix for ocean water", Appl. Opt. 23, 4427-4439 (1984).

8. Y. You, A. Tonizzo, A. A. Gilerson, M.E. Cummings, P. Brady, J. M. Sullivan, M. S. Twardowski, H. M. Dierssen, S. A. Ahmed, and G. W. Kattawar, "Measurements and simulations of polarization states of underwater light in clear oceanic waters”, Appl. Opt. 50, 4873-4893 (2011).

9. Y.Y. Schechner and N. Karpel, "Recovery of underwater visibility and structure by polarization analysis”, IEEE J. Ocean. Eng. 30, 570-587 (2005). 
10. P. C. Y. Chang, J. C. Flitton, K. I. Hopcraft, E. Jakeman, D. L. Jordan, and J. G. Walker, "Improving visibility depth in passive underwater imaging by use of polarization", Appl. Opt. 42, 2794-2803 (2003).

11. G. W. Kattawar and M. J. Rakovic, "Virtues of Mueller matrix imaging for underwater target detection”, Appl. Opt. 38, 6431-6438 (1999).

12. J. Cariou, B. Le Jeune, J. Lotrian, and Y. Guern, "Polarization effects of seawater and underwater targets", Appl. Opt. 29, 1689-1695 (1990).

13. G. D. Lewis, D. L. Jordan, and P. J. Roberts, "Backscattering target detection in a turbid medium by polarization discrimination”, Appl. Opt. 39, 3937-3944 (1999).

14. G. D. Gilbert and J. C. Pernicka, "Improvement of underwater visibility by reduction of backscatter with a circular polarization technique", Appl. Opt. 6, 741-746 (1967).

15. L. Bartolini, L. De Dominicis, G. Fornetti, M. Francucci, M. Guarneri, C. Poggi, and R. Ricci, "Improvement in underwater phase measurement of an amplitude-modulated laser beam by polarimetric techniques," Opt. Lett. 32, 1402-1404 (2007).

16. L. Mullen, B. Cochenour, W. Rabinovich, R. Mahon, and J. Muth, "Backscatter suppression for underwater modulating retroreflector links using polarization discrimination," Appl. Opt. 48, 328-337 (2009).

17. J. G. Walker, P. C. Y. Chang, and K. I. Hopcraft, "Visibility depth improvement in active polarization imaging in scattering media”, Appl. Opt. 39, 4933-4941 (2000).

18. D. A. Miller and E. L. Dereniak, "Selective polarization imager for contrast enhancements in remote scattering media”, App. Opt. 51, $4092-4102$ (2012).

19. M. Boffety, F. Galland, and A.G. Allais, "Influence of polarization filtering on image registration precision in underwater conditions”, Opt. Lett. 37, 3273-3275 (2012) 
20. T. Treibitz and Y. Y Schechner, “Active polarization descattering”, IEEE Trans. PAMI 31, 385-399 (2009).

21. J. Jaffe, "Computer modeling and the design of optimal underwater imaging systems", IEEE Ocean. Eng. 15, 101-110 (1990).

22. A. D. Kim and M. Moscoso, "Backscattering of circularly polarized pulses", Opt. Lett. 27, 1589-1591 (2002).

23. G. A. Gorman, "Field deployable dynamic lightning system for turbid water imaging”, M. S. Thesis, Massachusetts Institute of Technology, Cambridge - USA(2011).

24. P. C. Miller and R. S. Caprari, "Demonstration of improved automatic target-recognition performance by moment analysis of correlation peaks", Appl. Opt. 38, 1325-1331 (1999).

25. F. T. S Yu, D. A. Gregory, “Optical pattern recognition: architectures and techniques”, Proc. IEEE 84, 733-752 (1996).

26. A. Alfalou and C. Brosseau, "Understanding Correlation Techniques for Face Recognition: From Basics to Applications”, In Face Recognition, Milos Oravec (Ed.), ISBN: 978-953307-060-5, InTech, Available from: http://www.intechopen.com/articles/show/title/understanding-correlation-techniques-forface-recognition-from-basics-to-applications (2010)

27. I. Leonard, A. Alfalou, and C. Brosseau, "Spectral optimized asymmetric segmented phaseonly correlation filter”, Appl. Opt. 51, 2638-2650 (2012).

28. J. L. Horner, "Metrics for assessing pattern-recognition performance”, Appl. Opt. 31, 165166 (1992).

29. A. Alfalou, C. Brosseau, P. Katz, and M.S. Alam, "Decision optimization for face recognition based on an alternate correlation plane quantification metric", Opt. Lett. 37, 1562-1564 (2012). 
30. P. Katz, A. Alfalou, C. Brosseau, and M.S. Alam, "Correlation and independent component analysis based approaches for biometric recognition," in Face Recognition: Methods, Applications and Technology, edited by A. Quaglia and C. M. Epifano, Chap 11, pp.201229 (2012). ISBN: 978-1-61942-663-4

https://www.novapublishers.com/catalog/product_info.php?products_id=28370.

31. I. Leonard, A. Alfalou, and C. Brosseau, "Face recognition based on composite correlation filters: analysis of their performances", in Face Recognition: Methods, Applications and Technology, edited by A. Quaglia and C. M. Epifano, Chap 3, pp.57-80 (2012). ISBN: 978$1-61942-663-4$.

https://www.novapublishers.com/catalog/product_info.php?products_id=28370

32. Y. Ouerhani, M. Jridi and A. Alfalou, "Implementation techniques of high-order FFT into low-cost FPGA”, presented at the fifty fourth IEEE International Midwest Symposium on Circuits and Systems, Yonsei University, Seoul, Korea, 7-10 August 2011.

33. Y. Ouerhani, M. Jridi and A. Alfalou, "Fast Face Recognition Approach Using a Graphical Processing Unit "GPU". Proc. IEEE IST: International Conference on Imaging Systems and Techniques, 80-84 (2010).

34. A. VanderLugt, "Signal detection by complex spatial filtering", IEEE Trans. Inf. Theory, 10,139-145 (1964).

35. C. S. Weaver and J. W. Goodman, "A technique for optically convolving two functions", Appl. Opt. 5, 1248-1249 (1966).

36. Y. Piederrière, F. Boulvert, J. Cariou, B. Le Jeune, Y. Guern, and G. Le Brun, "Backscattered speckle size as a function of polarization: influence of particle-size and-concentration," Opt. Expr. 13, 5030-5039 (2005). 
37. P. Clemenceau, A Dogariu, and J Stryewski., "Polarization active imaging", Proc SPIE 4035, Laser Radar Technology and Applications V, 401-409, (2000)

38. J. S. Tyo, D. L. Goldstein, D. B. Chenault, and J. A. Shaw, "Review of passive imaging polarimetry for remote sensing applications”, Appl. Opt. 45, 5453-5469 (2006).

39. T. Kohlgraf-Owens and A. Dogariu, "Spatially resolved scattering polarimeter", Opt. Lett. 34, 1321, (2009) 


\section{Figure captions}

Fig.1: (Color online) Synoptic diagram of VLC architecture. FT denotes the Fourier transform.

Fig.2: Experimental setup for underwater target detection. Pol denotes a linear polarizer. QWP is a quarter wave plates. $\mathrm{L}_{1}, \mathrm{~L}_{2}, \mathrm{~L}_{3}, \mathrm{~L}_{4}$ are plano-convex convergent lenses. The detecting angle was set to $\theta=10^{\circ}$.

Fig.3: (Color online) Images of the plastic plug when 20ml of skimmed milk were added to 10L of tap water. (a) intensity image, (b) cross linear image, (c) signal estimation image and (d) clear water (no milk) image. The red square indicates the area where the degree of polarization of the backscatter was measured for target estimation. (e) Photograph of the plastic plug.

Fig.4: : (Color online) Correlation plane and PCE values $\left(\times 10^{4}\right)$ corresponding to the correlation of images displayed in Fig.3 with reference image (image in pure water). (a) intensity image I, (b) cross linear image (CRL), (c) target estimation image and (d) pure water (no milk) image.

Fig.5. (Color online) (a,d) PCE versus optical thickness $\tau_{0}$ for the plastic plug and different imaging modes showed in the inset. (b,e) Same as in (a,d) for the painted metal. (c,f) Same as in (a,d) for the rusted metal. The scattering medium is composed of skimmed milk (a,b,c) and semiskimmed milk (d,e,f) diluted in tap water.

Fig.6. Degree of polarization of the backscatter $\left(p_{\text {scat }}\right)$ versus optical thickness for experiments in skimmed milk.

Fig.7. (Color online) (a) PCE as a function of target depth for painted metal in ocean water. (b) Same as in (a) for lake water. 


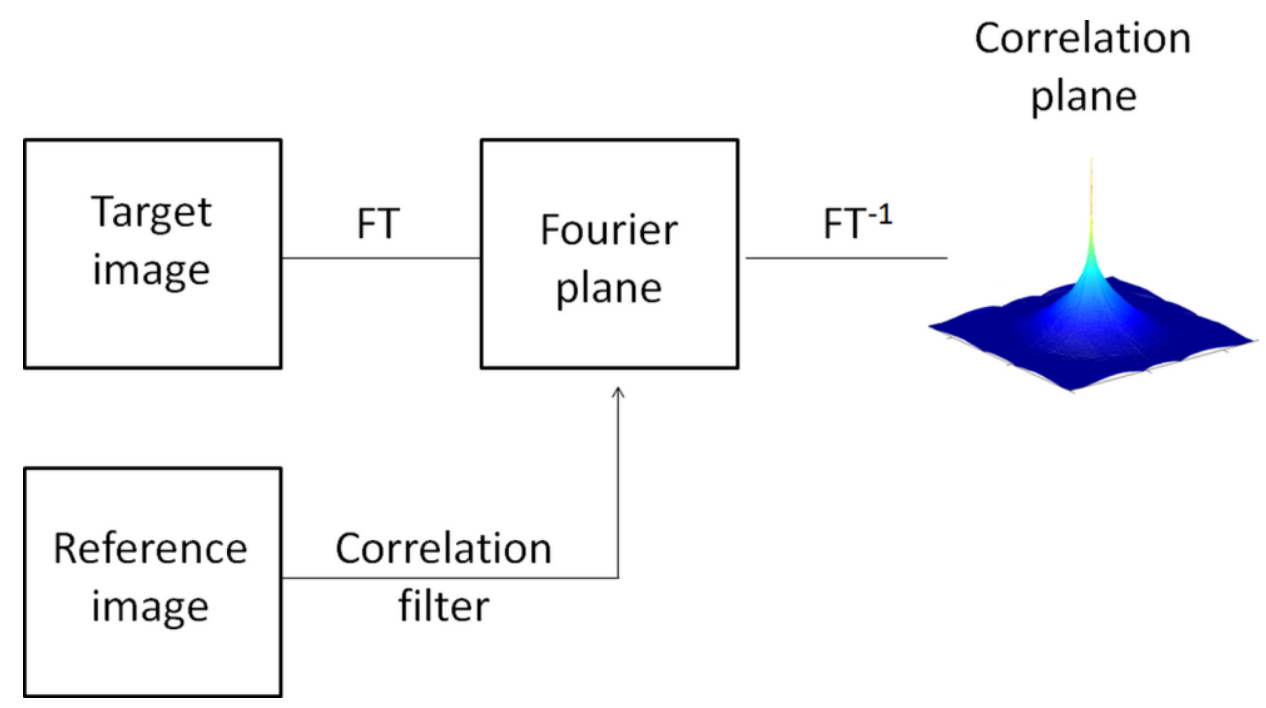

Fig.1: (Color online) Synoptic diagram of VLC architecture. FT denotes the Fourier transform. 


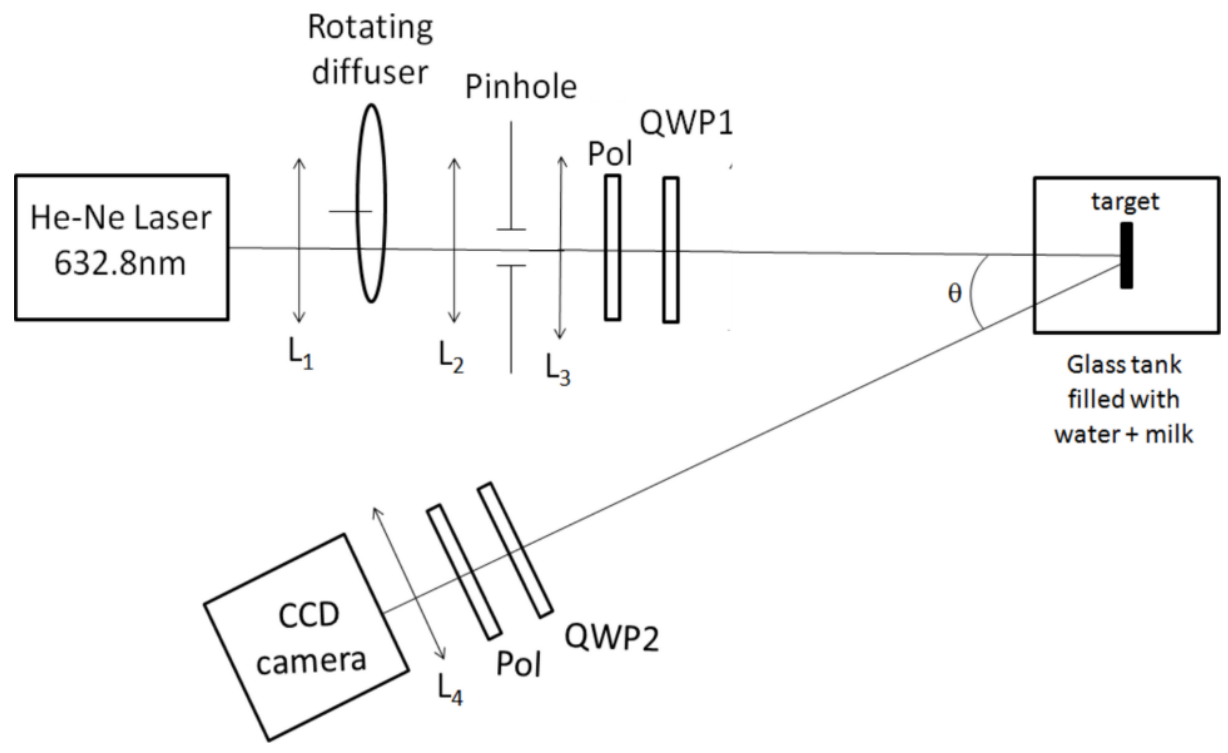

Fig.2: Experimental setup for underwater target detection. Pol denotes a linear polarizer. QWP is a quarter wave plates. $\mathrm{L}_{1}, \mathrm{~L}_{2}, \mathrm{~L}_{3}, \mathrm{~L}_{4}$ are plano-convex convergent lenses. The detecting angle was set to $\theta=10^{\circ}$. 
(a)
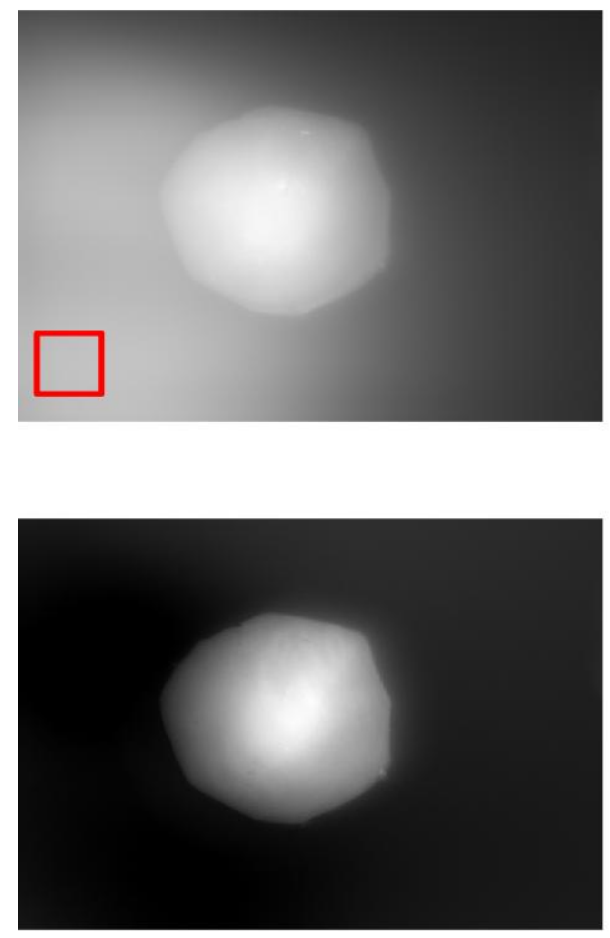

(c) (b)
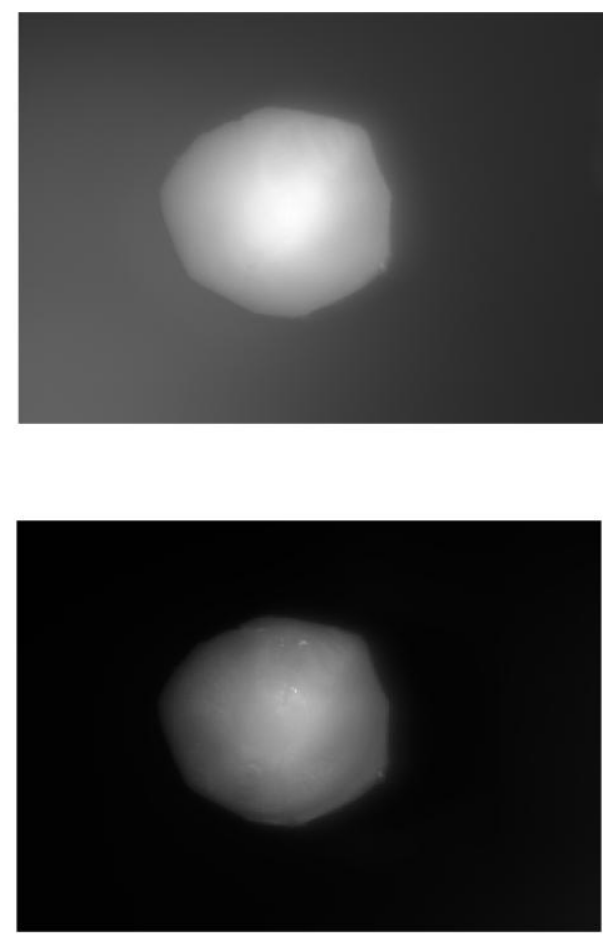

(d)

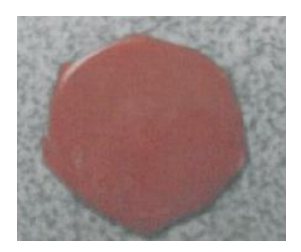

(e)

Fig.3: (Color online) Images of the plastic plug when $20 \mathrm{ml}$ of skimmed milk were added to $10 \mathrm{~L}$ of tap water. (a) intensity image, (b) cross linear image, (c) signal estimation image and (d) clear water (no milk) image. The red square indicates the area where the degree of polarization of the backscatter was measured for target estimation. (e) Photograph of the plastic plug. 
(a)

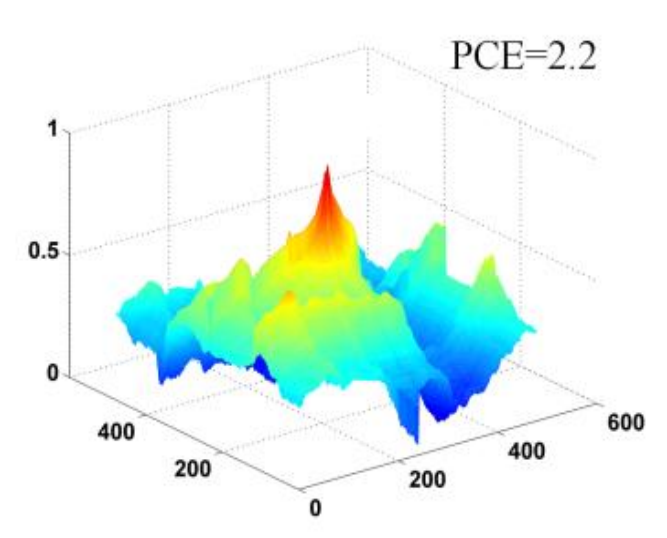

(c)

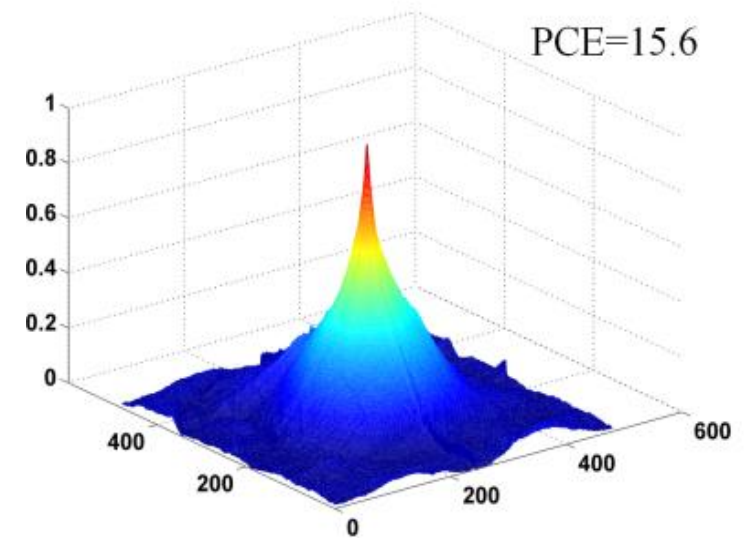

(b)

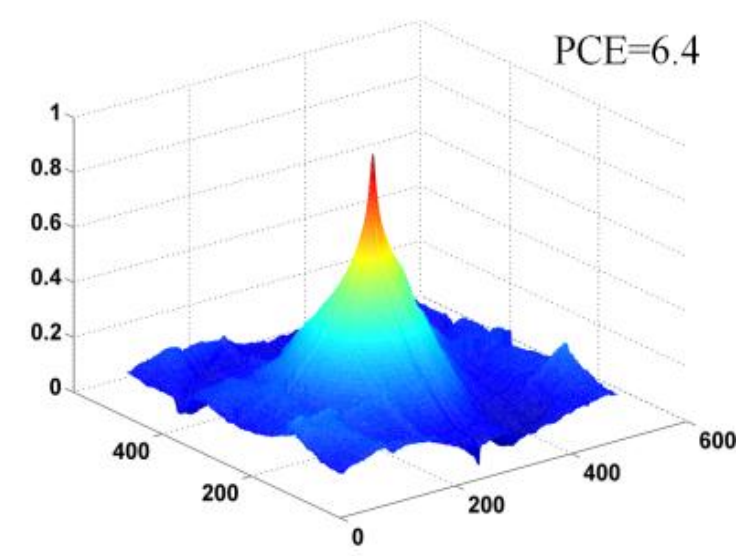

(d)

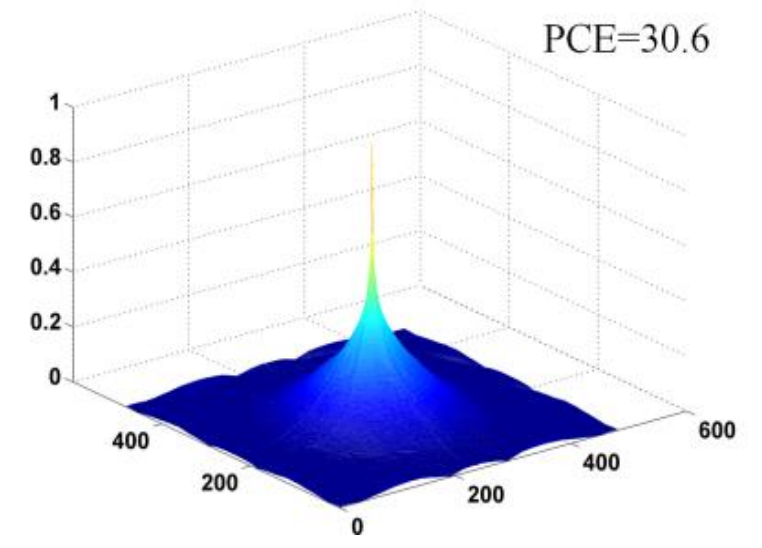

Fig.4: (Color online) Correlation plane and PCE values $\left(\times 10^{4}\right)$ corresponding to the correlation of images displayed in Fig.3 with reference image (image in pure water). (a) intensity image I, (b) cross linear image (CRL), (c) target estimation image and (d) pure water (no milk) image. 
(a)

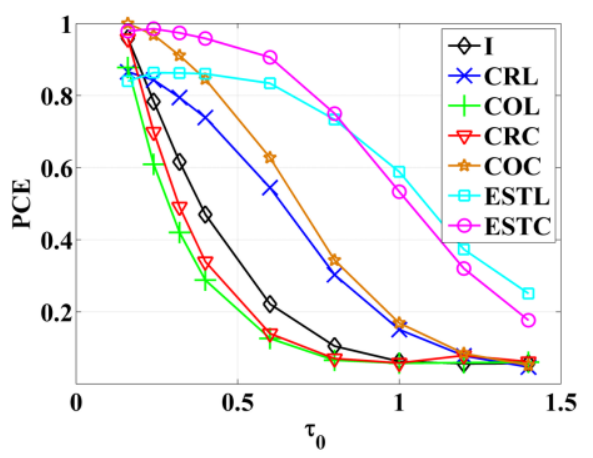

(b)

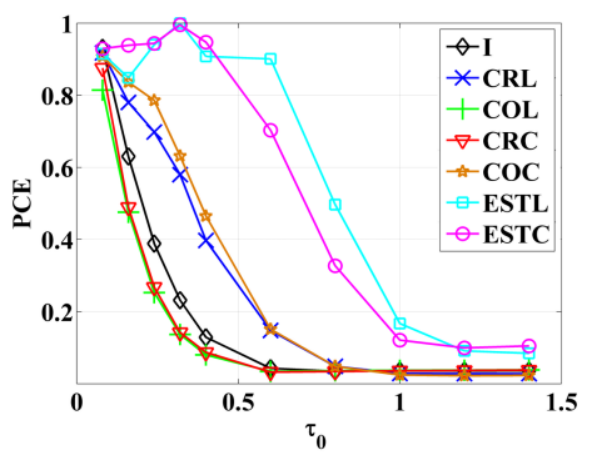

(c)

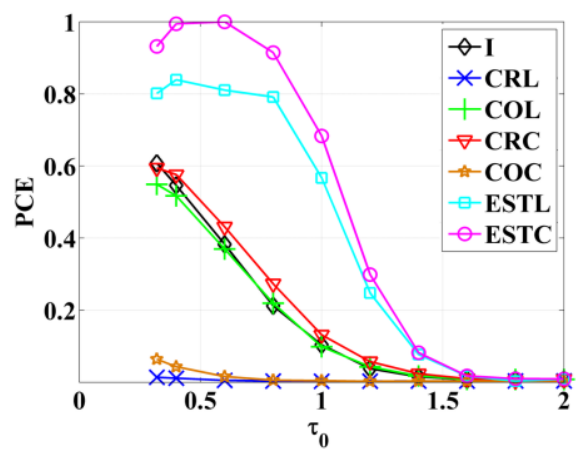

(d)

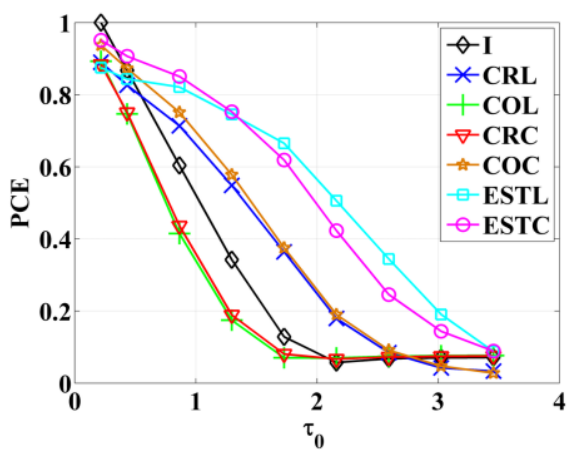

(e)

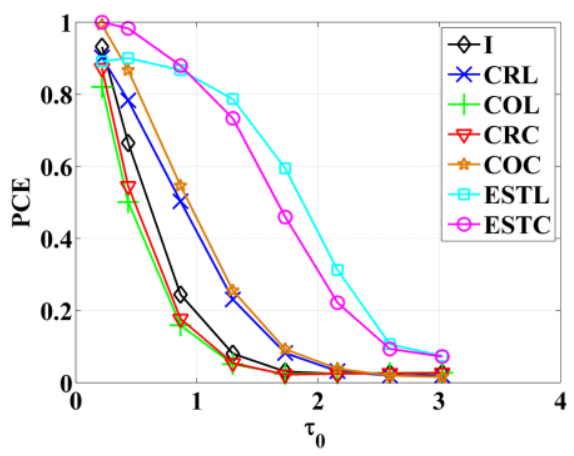

(f)

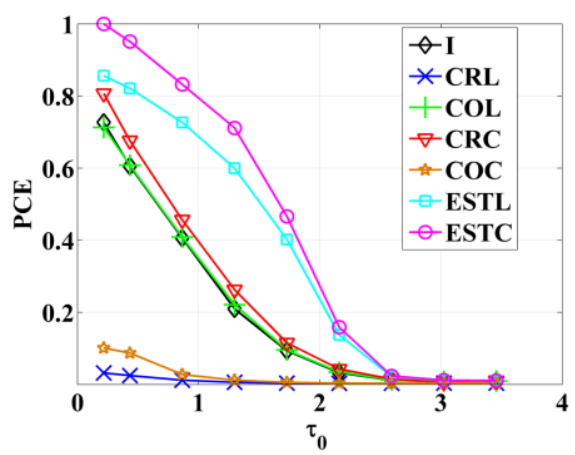

Fig.5. (Color online) (a,d) PCE versus optical thickness $\tau_{0}$ for the plastic plug and different imaging modes showed in the inset. (b,e) Same as in (a,d) for the painted metal. (c,f) Same as in $(\mathrm{a}, \mathrm{d})$ for the rusted metal. The scattering medium is composed of skimmed milk $(\mathrm{a}, \mathrm{b}, \mathrm{c})$ and semiskimmed milk $(\mathrm{d}, \mathrm{e}, \mathrm{f})$ diluted in tap water. 


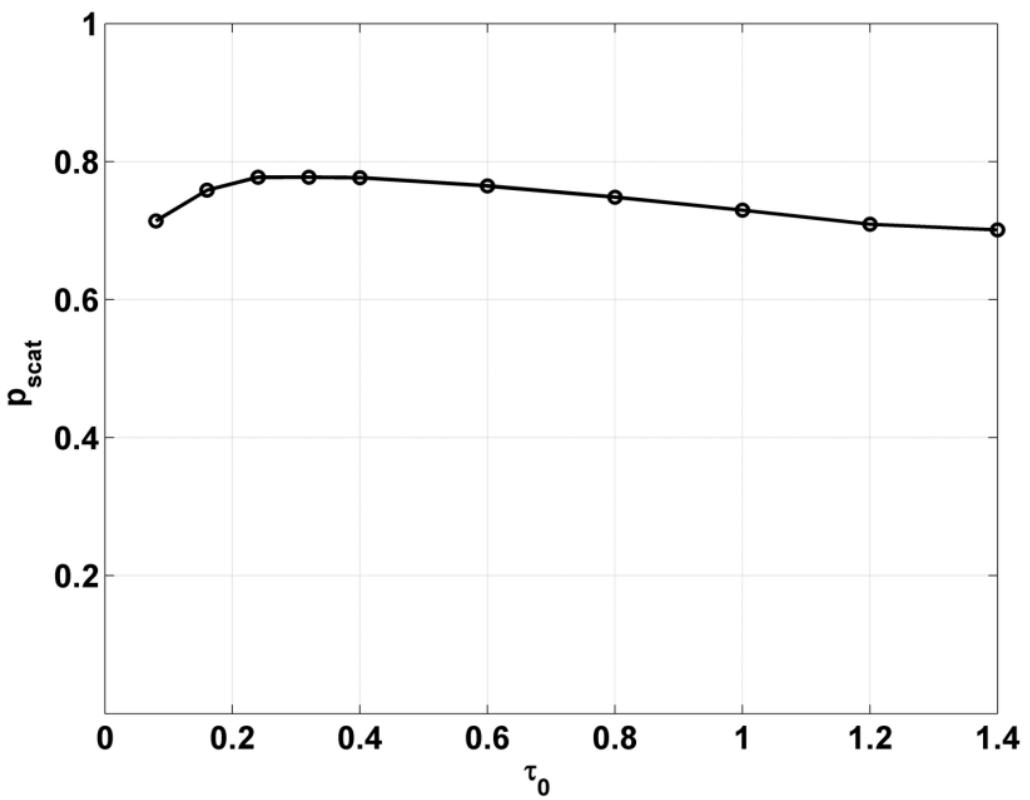

Fig.6. Degree of polarization of the backscatter $\left(p_{\text {scat }}\right)$ versus optical thickness for experiments in skimmed milk. 
(a)

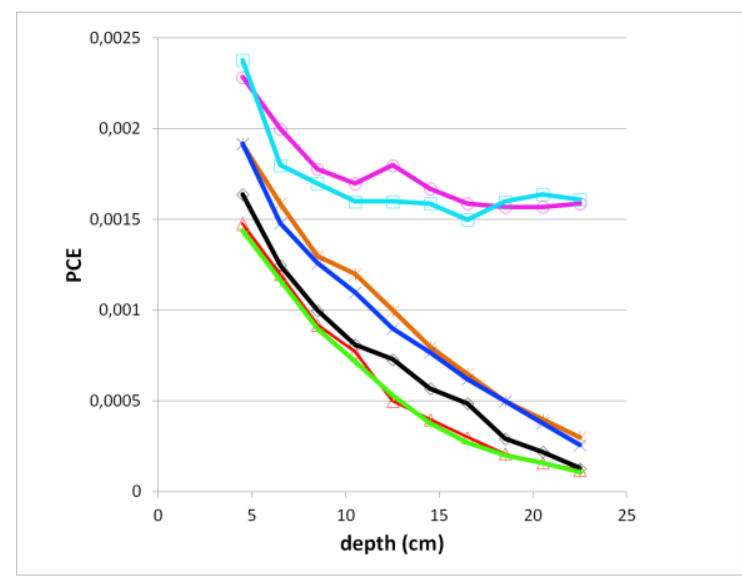

(b)

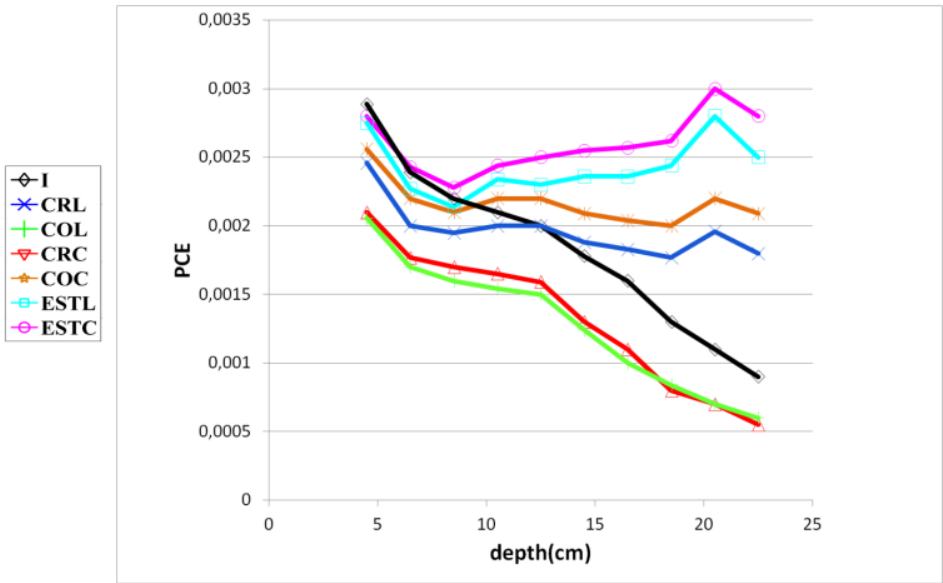

Fig.7. (Color online) (a) PCE as a function of target depth for painted metal in ocean water. (b) Same as in (a) for lake water. 